\title{
An increase in early cancer detection rates at a single cancer center: Experiences from Sun Yat-sen University Cancer Center
}

\author{
Shang-Hang Xie ${ }^{1}$, Ri-Qing Huang ${ }^{2}$, Yi-Long Liu ${ }^{1}$, Su-Mei Cao ${ }^{1, *}$, and Chao-Nan Qian ${ }^{3,4, *}$ \\ ${ }^{1}$ Department of Epidemiology, Collaborative Innovation Center for Cancer Medicine, Sun Yat-sen University Cancer Center, \\ Guangzhou 510060, China \\ 2 Department of Medical Oncology, Guangzhou Concord Cancer Center, Guangzhou 510555, China \\ 3 State Key Laboratory of Oncology in South China, Collaborative Innovation Center for Cancer Medicine, \\ Sun Yat-sen University Cancer Center, Guangzhou 510060, China \\ 4 Department of Radiation Oncology, Guangzhou Concord Cancer Center, Guangzhou 510555, China
}

Received 23 November 2021, Accepted 7 February 2022, Published online 1 March 2022

\begin{abstract}
Cancer has become a major fatal disease in China. The relatively lower early detection rates for multiple cancer types have been one of the main reasons for a relatively lower cancer curative rate in China compared with the developed countries. To investigate trends in the early cancer detection rate over the past 5 years in a major city of China, 45,260 patients with newly diagnosed cancers of the nasopharynx, lung, thyroid, colorectum, liver, breast, uteral cervix, stomach, esophagus, blood, and kidney from 2016 to 2020 at Sun Yat-sen University Cancer Center were evaluated. The early detection rate (stage I disease) for all cancer types in combination significantly increased from 14.4 to $23.07 \%$. Among the studied cancer types, a significant increase in stage I cancers was proportionally seen in cancers of the lung, thyroid, colorectum, and uterine cervix. While for cancers of the liver and stomach, a significant proportional increment was only observed when combining stage I and stage II diseases. No significant alteration in early cancer detection of the nasopharynx, breast, esophagus, blood, or kidney was observed. Three limitations of this present study include relatively small cohorts of cancer patients, relatively short observation periods, and limited sample representativeness. Further efforts are anticipated to validate our findings with larger patient cohorts from different parts of China and enhance early cancer detection rates by promoting public awareness, applying better health care policies, and improving insurance coverage and medical resources.
\end{abstract}

Key words: Cancer incidence rate, Early detection, Lung cancer, Thyroid cancer, Nasopharyngeal cancer, Colorectal cancer, Gastric cancer, Cervical cancer, Renal cancer.

\section{Background}

Cancer has become a major fatal disease in China, with over 3.9 million yearly new cases and over 2.3 million cancer deaths [1]. One of the key factors responsible for the high mortality rate of cancer patients in China is a relatively low early detection rate [2].

Different cancers have different alarm symptoms. For those with apparent early alarm symptoms, the proportion of stage I disease is expected to be high; and this is the rationale for promoting public awareness. With improvements in public awareness, insurance coverage, and the healthcare system, early cancer detection rates are expected to improve in most of China [3-5]. However, the available data for analyzing early detection rates in China is limited, not to mention the dynamic alteration of early detection rates with the passage of time. In this present study, we retrieved data of newly diagnosed cancer patients from a single institution in a major Chinese city to generate some clues to better understand the increase in early cancer detection rates in China, followed by a discussion on the possible underlying and influencing factors.

\section{Methods and materials}

\section{Patient cohorts}

Patients with newly diagnosed cancers of the nasopharynx, lung, thyroid, colorectum, liver, breast, uteral cervix, stomach, esophagus, blood, and kidney from 2016 to 2020 at Sun Yat-sen University Cancer Center were analyzed. These patients had sufficient clinicopathological data for staging analysis. The total analyzed patient numbers were 8,601 in 2016, 8,185 in $2017,8,565$ in $2018,9,573$ in 2019 , and 10,336 in

\footnotetext{
*Corresponding authors: caosm@sysucc.org.cn; qianchn@sysucc.org.cn 
$\square$ Stage I $\square$ Stage II $\square$ Stage III $\square$ Stage IV

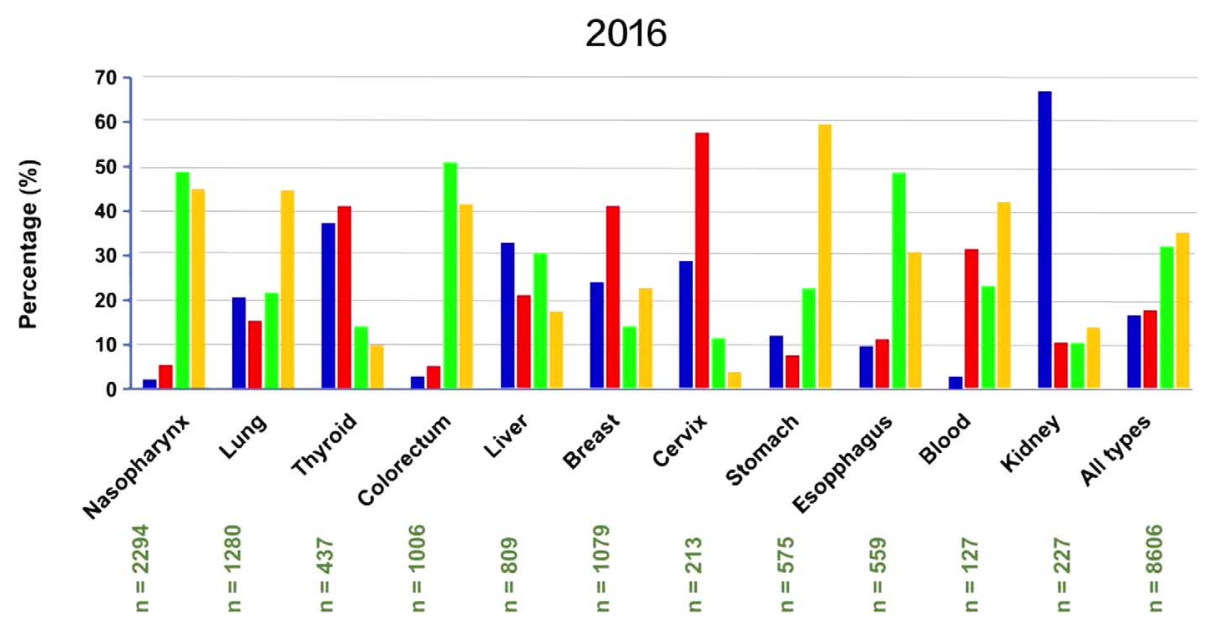

Video 1. Dynamic alterations of early detection rates among the 11 most commonly seen malignancies at SYSUCC between 2016 and 2020. Increments in early detection rates for lung, thyroid, colorectum, cervix, liver, and stomach cancer resulted in a steady increase in early detection rates for all types of cancer when combined together. https://vcm.edpsciences.org/10.1051/vcm/2022001\#V1

2020. This present study was approved by the Institutional Review Board of Sun Yat-sen University Cancer Center with the approval number: ZDMB-2020-001-01.

\section{The staging systems}

The 8th edition American Joint Committee on Cancer (AJCC) TNM staging systems were used for nasopharyngeal cancer, breast cancer, gastric cancer, cervical cancer, thyroid cancer, colorectal cancer, hepatocellular cancer, and renal cancer. The 8th edition of the Union for International Cancer Control (UICC) staging systems were used for lung cancer and esophageal cancer. Blood cancers in this present study included lymphoma (staged using the Ann Arbor MIPI, and LUGANO systems), leukemia (staged using the Rai system), and multiple myeloma (staged using the Revised International Staging (R-ISS) system).

\section{Statistical analyses}

The Cochran-Armitage trend test was applied to evaluate the percentage increments in early-stage diseases. A $P$ value less than 0.05 was considered significant.

\section{Results}

\section{General tendency}

Video 1 shows the proportional alterations of four clinical stages for different cancer types within the studied 5-year period. Generally speaking, the early detection rate for all cancer types calculated by stage I tumors alone significantly increased from 14.4 to $23.07 \%$ within the 5 years (Table 1 ).
Increase in early cancer detection rates of the lung, thyroid, colorectum, cervix, liver, and stomach

Table 1 shows a significant increase in the proportion of stage I cancers of the lung, thyroid, colorectum, and uterine cervix. While in the liver and stomach cancers, the significant proportional increase was only observed by combining stage I and stage II cancers.

\section{No significant changes in early cancer detection of the nasopharynx, breast, esophagus, blood, or kidney}

Even though nasopharyngeal carcinoma (NPC) is a common malignancy in southern China [6], its early detection rate is extremely low (1.6-2.5\% of stage I disease). Notably, the relatively high early detection rates in kidney cancer (57.7-66.7\% for stage I disease) were not significantly altered within the studied time period, neither were early cancer detection rates for breast (18.2-24.3\%), esophagus (9.4-11.7\%), or blood $(0-7.3 \%)$.

\section{Discussion}

The benefits of promoting early cancer detection and early cancer treatment include saving more lives, reducing direct medical costs, reducing the disability rate, reducing personal psychological stress, and preserving patients' vitality. Two major factors influencing China's early cancer detection rate are medical insurance coverage and public awareness of early cancer detection [7, 8]. However, it is also believed that limited medical resources for routine medical check-ups among the adult population in China have also prevented early detection for most cancer types.

Lung cancer has been the leading cause of cancer-related deaths in many countries for many years, with its incidence 
Table 1. Increase in early detection rates presented by the percentages of stage I or stages I + II diseases.

\begin{tabular}{|c|c|c|c|c|c|c|c|}
\hline \multirow[t]{2}{*}{ Cancer Type } & \multirow[t]{2}{*}{ Stage included } & \multicolumn{5}{|c|}{ Percentage in indicated year } & \multirow{2}{*}{$\begin{array}{c}\text { Cochran-Armitage }(Z) \\
P \text { Value }\end{array}$} \\
\hline & & 2016 & 2017 & 2018 & 2019 & 2020 & \\
\hline Lung & Stage I & 16.88 & 20.24 & 25.29 & 31.94 & 36.17 & $<0.0001$ \\
\hline Thyroid & Stage I & 29.98 & 37.63 & 45.95 & 41.29 & 56.87 & $<0.0001$ \\
\hline Colorectum & Stage I & 4.47 & 2.79 & 6.74 & 5.92 & 6.59 & 0.0031 \\
\hline Cervix & Stage I & 10.33 & 30.26 & 33.54 & 23 & 37.94 & $<0.0001$ \\
\hline Liver & Stages I and II & 54.51 & 52.51 & 60.37 & 58.55 & 61.04 & 0.0007 \\
\hline Stomach & Stages I and II & 16.35 & 19.23 & 16.97 & 27.23 & 29.48 & $<0.0001$ \\
\hline All types & Stage I & 14.4 & 16.48 & 20.27 & 18.49 & 23.07 & $<0.0001$ \\
\hline
\end{tabular}

highly related to smoking practice [9]. The incidence of lung cancer in the male population of China has been stable at about $47 / 100,000$ between the years 2000 and 2015, while it has increased in females from $20 / 100,000$ to $25 / 100,000$ for the time period between 2010 and 2015 [1]. Although this deadly disease had a very high mortality rate before the emerging and wide application of targeted drug therapy, a slight reduction in lung cancer mortality in both genders between 2000 and 2015 has been observed in China [1]. This can be explained by increased public awareness and an increase in low-dose computed tomography (CT) scanning among cigarette smokers [10].

Despite increases in lung cancer incidence in both genders in China over the past several decades [11, 12], the early detection rate of lung cancer has also been improved in several institutions $[13,14]$. It is expected that in addition to using low-dose CT for screening, the application of other novel self-sampling technologies might be able to further improve the lung cancer early detection rate [2].

Thyroid cancer is the most common head-and-neck malignancy globally and accounts for 1-2\% of all malignancies worldwide [15]. The rapid increase in the incidence of thyroid cancer has been reported by other researchers $[16,17]$. In 2015 , approximately 200,700 new thyroid cancer cases were diagnosed in China, accounting for $5.11 \%$ of all new cancer cases, with the incidence rate ranked 7 th among all cancers [17]. The major histological types of thyroid cancer include papillary thyroid carcinoma (PTC), follicular thyroid carcinoma (FTC), medullary thyroid carcinoma (MTC), and poorly differentiated thyroid cancer.

Both papillary and follicular cancers derive from thyroid follicular epithelial cells, and they are grouped as differentiated thyroid cancer (DTC), accounting for $95 \%$ of cases. With total thyroidectomy and radioiodine treatment, DTC is an indolent and curable disease in most patients, whereas the curative rate of MTC is lower and depends on early diagnosis. The histologic type of thyroid cancer which has contributed to a rapid increase in its worldwide incidence is PTC [18]. This rapid increase in thyroid cancer has aroused widespread public concern. Environmental risk factors, including a deficit or excess of iodine intake [19], medical radiation [20], and nutritionrelated factors [21], have also been proposed to be partially responsible for this increase. The application of advanced diagnostic techniques, especially ultrasonography, computed tomography, and magnetic resonance imaging, have been proposed as the main reason for the increase in early detection rates [22-24].

The greatest potential for reducing cancer-related deaths is through increasing early diagnosis rates in the short term. This understanding has led to efforts to detect symptomatic cancers earlier and, eventually, to detect occult cancers in asymptomatic persons through screening. From the mid-20th century, accumulating evidence has supported the introduction of screening for cancers of the cervix, breast, colorectum, prostate, and lung. Screening for cancer using radiographic imaging has been available for decades, and multiple clinical studies have demonstrated its efficacy in specific instances. The three cancers with the best consensus for the greatest benefits from early cancer detection using imaging include breast cancer, colorectal cancer, and lung cancer. Blood-based tests often called liquid biopsies, which detect circulating tumor-derived factors such as circulating tumor cells (CTCs) [25] and circulating tumor DNA (ctDNA) [26], have shown potential usage in early diagnosis. ctDNA testing could also be used synergistically with other multi-omic biomarkers to enhance early detection. For instance, assays that incorporate early detection proteins (i.e., CA-125), epigenetic markers, circulating tumor RNA, nucleosomes, exosomes, and associated immune markers have been explored [27]. However, the sensitivity of these tests to detect small tumors is still too low for routine clinical usage. Many obstacles must be overcome before a blood-based screening test, incorporating either CTCs or ctDNA, could become a valuable asset for early cancer diagnosis.

There are several other barriers preventing early cancer detection when screening high-risk populations. Individuals with lower economic incomes [28], lower educational achievements [29], and those without insurance [28, 30] are less likely to join screening programs. With improvements in health care policies and insurance coverage, it is expected that more high-risk populations might be willing to join cancer screening initiatives [31-33].

Three limitations of this present study include relatively small cohorts of cancer patients, relatively short observation periods, and limited sample representativeness as all data came from a single institution. These limitations might generate biases in our findings. Therefore, our findings should be validated by further studies with larger cohorts from different parts of China with longer observation periods.

In conclusion, despite the limited representativeness, increments of early detection rates of multiple cancer types have 
been observed over the past 5 years at a single institution in South China. More efforts are expected for further enhancing early cancer detection rates by improvements in public awareness, health care policy, insurance coverage, and medical resources.

\section{Conflict of interest}

Authors declare that they have no competing interests.

Acknowledgements. This work was supported by grants to ChaoNan Qian from the National Natural Science Foundation of China (No. 82073220, No. 81872384, and No. 81672872).

\section{References}

1. Zhang S, Sun K, Zheng R, et al. Cancer incidence and mortality in China, 2015. Journal of the National Cancer Center 2021;1(1):2-11.

2. Qian CN. At-home cancer screening: a solution for China and other developing countries with a large population and limited number of healthcare practitioners. Chinese Journal of Cancer. 2017;36(1):68.

3. Chen W, Zheng R, Baade PD, et al. Cancer statistics in China 2015. CA: A Cancer Journal for Clinicians. 2016;66(2):115-132.

4. Chen W, Zheng R, Zhang S, et al. Cancer incidence and mortality in China, 2013. Cancer Letters. 2017;401:63-71.

5. Zeng $\mathrm{H}$, Chen $\mathrm{W}$, Zheng $\mathrm{R}$, et al. Changing cancer survival in China during 2003-15: a pooled analysis of 17 population-based cancer registries. Lancet Glob Health. 2018;6(5):e555-e567.

6. Guo LL, Wang HY, Zheng LS, et al. Metastasis of nasopharyngeal carcinoma: What we know and do not know. Visualized Cancer Medicine. 2021;2:1-13.

7. Feng RM, Zong YN, Cao SM, et al. Current cancer situation in China: Good or bad news from the 2018 Global Cancer Statistics? Cancer Communications. 2019;39(1):22.

8. Sun D, Li H, Cao M, et al. Cancer burden in China: trends, risk factors and prevention. Cancer Biology \& Medicine. 2020; 17(4):879-895.

9. Barta JA, Powell CA, Wisnivesky JP, Global epidemiology of lung cancer. Annals of Global Health. 2019;85(1):100-202.

10. Wood DE, Kazerooni EA, Baum SL, et al. Lung Cancer Screening, Version 3.2018, NCCN Clinical Practice Guidelines in Oncology. Journal of the National Comprehensive Cancer Network. 2018;16(4):412-441.

11. Cao M, Chen W. Epidemiology of lung cancer in China. Thoracic Cancer. 2019;10(1):3-7.

12. He J, Li N, Chen W, et al. China guideline for the screening and early detection of lung cancer (2021 Beijing). Zhonghua Zhong Liu Za Zhi. 2021;43(3):243-268.

13. Guo LW, Liu SZ, Zhang SK, et al. Analysis of the efficacy of lung cancer screening in urban areas of Henan Province by lowdose computed tomography from 2013 to 2017. Zhonghua zhong liu za zhi [Chinese Journal of Oncology]. 2020;42(2): $155-159$.

14. Zhao S, Wu N. Early detection of lung cancer: Low-dose computed tomography screening in China. Thoracic Cancer. 2015;6(4):385-389.

15. Lawal O, Agbakwuru A, Olayinka OS, et al. Thyroid malignancy in endemic nodular goitres: prevalence, pattern and treatment. European Journal of Surgical Oncology: The Journal of the European Society of Surgical Oncology and the British Association of Surgical Oncology. 2001;27(2):157-161.

16. Du L, Wang Y, Sun $X$, et al. Thyroid cancer: trends in incidence, mortality and clinical-pathological patterns in Zhejiang Province, Southeast China. BMC Cancer. 2018; 18(1):291.

17. Du L, Zhao Z, Zheng R, et al. Epidemiology of thyroid cancer: Incidence and mortality in China, 2015. Frontiers in Oncology. 2020;10:1702.

18. Davies L, Welch HG. Current thyroid cancer trends in the United States. JAMA Otolaryngology - Head and Neck Surgery. 2014;140(4):317-322.

19. Dal Maso L, Bosetti C, La Vecchia C, et al. Risk factors for thyroid cancer: an epidemiological review focused on nutritional factors. Cancer Causes and Control: CCC. 2009;20(1): $75-86$.

20. Schonfeld SJ, Lee C, Berrington de González A. Medical exposure to radiation and thyroid cancer. Clinical Oncology (Royal College of Radiologists (Great Britain)). 2011;23(4): 244-250.

21. Cho YA, Kim J. Dietary factors affecting thyroid cancer risk: A meta-analysis. Nutrition and Cancer. 2015;67(5):811-817.

22. Brito JP, Al Nofal A, Montori VM, et al. The impact of subclinical disease and mechanism of detection on the rise in thyroid cancer incidence: a population-based study in Olmsted County, Minnesota during 1935 through 2012. Thyroid: Official Journal of the American Thyroid Association. 2015;25(9):1-2.

23. O'Grady TJ, Gates MA, Boscoe FP. Thyroid cancer incidence attributable to overdiagnosis in the United States 1981-2011. International Journal of Cancer. 2015;137(11):2664-2673.

24. Vaccarella S, Dal Maso L, Laversanne M, et al. The impact of diagnostic changes on the rise in thyroid cancer incidence: a population-based study in selected high-resource countries. Thyroid: Official Journal of the American Thyroid Association. 2015;25(10):1127-1136.

25. Poudineh M, Sargent EH, Pantel K, et al. Profiling circulating tumour cells and other biomarkers of invasive cancers. Nature Biomedical Engineering. 2018;2(2):72-84.

26. Cai X, Janku F, Zhan Q, et al. Accessing genetic information with liquid biopsies. Trends in Genetics: TIG. 2015;31(10): 564-575.

27. Campos-Carrillo A, Weitzel JN, Sahoo P, et al. Circulating tumor DNA as an early cancer detection tool. Pharmacology \& Therapeutics. 2020;207:107458.

28. Sabatino SA, White MC, Thompson TD, et al. Cancer screening test use - United States, 2013. MMWR Morbidity and Mortality Weekly Report. 2015;64(17):464-468.

29. Damiani G, Basso D, Acampora A, et al. The impact of level of education on adherence to breast and cervical cancer screening: Evidence from a systematic review and meta-analysis. Preventive Medicine. 2015;81:281-289.

30. Carney PA, O'Malley J, Buckley DI, et al. Influence of health insurance coverage on breast, cervical, and colorectal cancer screening in rural primary care settings. Cancer. 2012;118(24): 6217-6225.

31. Sabatino SA, Lawrence B, Elder R, et al. Effectiveness of interventions to increase screening for breast, cervical, and colorectal cancers: nine updated systematic reviews for the guide to community preventive services. American Journal of Preventive Medicine. 2012;43(1):97-118. 
32. Stange KC, Breslau ES, Dietrich AJ, et al. State-of-the-art and future directions in multilevel interventions across the cancer control continuum. Journal of the National Cancer Institute Monographs. 2012;2012(44):20-31.
33. Taplin SH, Anhang Price R, Edwards HM, et al. Introduction: Understanding and influencing multilevel factors across the cancer care continuum. Journal of the National Cancer Institute Monographs. 2012;2012(44):2-10.

Cite this article as: Xie S-H, Huang R-Q, Liu Y-L, Cao S-M, \& Qian C-N. An increase in early cancer detection rates at a single cancer center: Experiences from Sun Yat-sen University Cancer Center. Visualized Cancer Medicine. 2022. 3, 1. 